\title{
Membrane-catalyzed IAPP aggregation is dominated by secondary nucleation
}

Barend O.W. Elenbaas ${ }^{1}$, Lucie Khemtemourian ${ }^{2}$, J. Antoinette Killian ${ }^{1}$, Tessa Sinnige ${ }^{1, *}$

1. Membrane Biochemistry and Biophysics, Bijvoet Centre for Biomolecular Research, Utrecht University, Padualaan 8, $3584 \mathrm{CH}$, Utrecht, The Netherlands

2. Institute of Chemistry \& Biology of Membranes \& Nanoobjects (CBMN), CNRS UMR5248, University of Bordeaux, Bordeaux INP, allée Geoffroy St-Hilaire, 33600, Pessac, France

*corresponding author, t.sinnige1@uu.nl

\section{Abstract}

Type II diabetes is characterized by the loss of pancreatic $\beta$-cells, which produce insulin. $\beta$-Cell death is thought to be a consequence of membrane disruption, caused by the aggregation of islet amyloid polypeptide (IAPP) into amyloid fibrils. However, the molecular mechanisms of IAPP aggregation in the presence of membranes have remained unclear. Global fitting of kinetic data has proven to be a valuable method to reveal the microscopic steps of amyloid formation. Here, we use this methodology to elucidate the aggregation mechanism of IAPP in the presence of mixed zwitterionic and anionic lipid membranes. The results of our kinetic analysis converge to a previously undescribed model, in which aggregation on the membrane is strongly dominated by secondary nucleation, i.e. the formation of new nuclei on the surface of existing fibrils. The critical nucleus consists of a single IAPP molecule, and multiple anionic lipids are involved in catalyzing both primary and secondary nucleation. The elongation rate does not appear to be affected by the presence of anionic lipids. These new insights into the mechanism of IAPP aggregation on membranes may help to understand IAPP toxicity and will be important for the development of therapeutics to prevent $\beta$-cell death in type II diabetes.

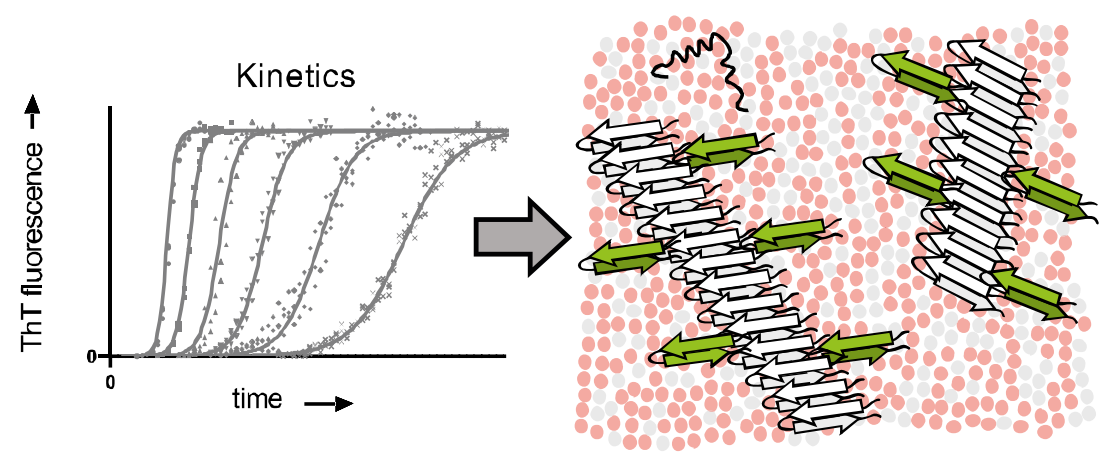


Type II diabetes is a major health problem affecting about 500 million people worldwide ${ }^{1,2}$. A major contributor to the pathophysiology of the disease is the loss of $\beta$-cells in the pancreas ${ }^{3,4}$. This loss has been attributed to the aggregation of islet amyloid polypeptide (IAPP), a 37 amino acid-long hormone with various functions that is co-secreted with insulin from the $\beta$-cells. The process of fibril formation by IAPP is thought to disturb the membranes of the $\beta$-cells, which ultimately kills them ${ }^{5,6}$.

Monomeric IAPP interacts with membranes, and in particular anionic lipids have been shown to accelerate fibril formation of the peptide ${ }^{7-11}$. However, mechanistic insights into IAPP aggregation have thus far been restricted to solution conditions in the absence of lipid membranes. Several studies have demonstrated that secondary nucleation plays a dominant role in IAPP aggregation in solution, meaning that the fibril surface mediates the formation of new nuclei once initial fibrils have formed ${ }^{12-}$ 14. Recently, this was confirmed by a quantitative analysis using global fitting of Thioflavin T (ThT) kinetic data to mathematical models of amyloid formation ${ }^{14}$, which is a powerful tool to establish aggregation mechanisms ${ }^{15-17}$. Here, we employ this approach to elucidate the molecular mechanisms of IAPP aggregation in the presence of lipid membranes, based on high quality ThT data for synthetic human IAPP (Fig. S1).

In order to establish the contribution of lipid membranes to the aggregation kinetics of IAPP, we first performed the aggregation assay in the absence of membranes. Under these conditions, the kinetics of IAPP aggregation are not concentration-dependent as can be seen from the overlap between the normalized ThT curves (Fig. 1A). These results are in agreement with previous studies using synthetic peptide that attributed this behavior to the existence of IAPP condensates or micelles, resulting in a constant aggregation rate that is independent of the bulk monomer concentration ${ }^{12,18}$. Similar findings were recently reported for another amyloid-forming protein, $\alpha$-synuclein, in its phase-separated state ${ }^{19}$.

A different view emerges when looking at IAPP aggregation kinetics in the presence of large unilamellar vesicles (LUVs) composed of zwitterionic and anionic lipids (1,2-dioleoyl-sn-glycero-3-phosphocholine, DOPC, and 1,2-dioleoyl-sn-glycero-3-phospho-L-serine, DOPS, respectively) in a molar ratio of 7:3. At peptide: total lipid molar ratios ranging from 1:10 to 1:100, the aggregation kinetics become faster with increasing IAPP monomer concentration (Fig 1B-D). To assess the concentration dependence, a double-log plot was created, in which the logarithm of the initial monomer concentration $\left(m_{0}\right)$ is plotted against that of the half-time (timepoint at which half the maximum fluorescence intensity is reached, $\left.t_{1 / 2}\right)^{17}$. For all three IAPP:lipid ratios examined, the datapoints lie on a straight line with a slope of approximately -0.9 to -1.0 , which is the value of the scaling exponent $(\gamma)$ (Fig. 1E, F). By contrast, in the absence of lipids, the scaling exponent of IAPP is close to 0 , reflecting the lack of concentration dependence (Fig. 1A, E, F). 


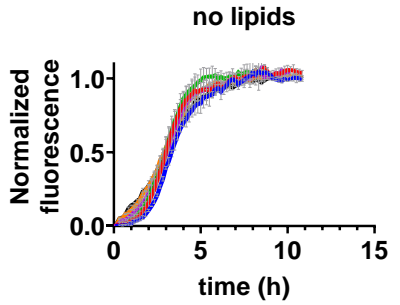

C

$1: 50$

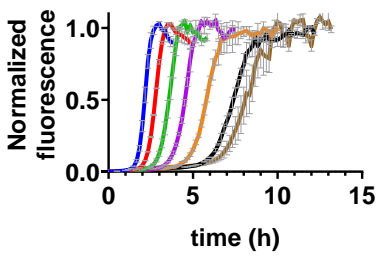

E

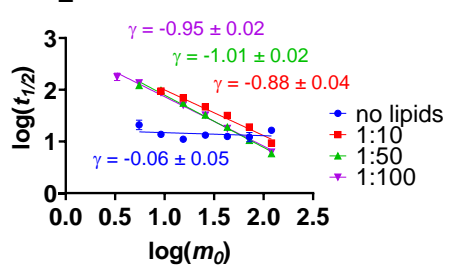

B

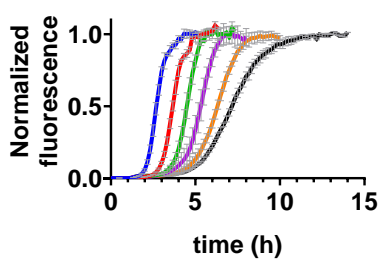

D

$1: 100$

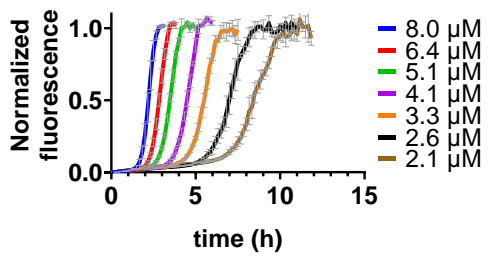

$\mathrm{F}$

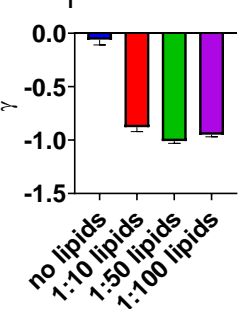

Figure 1) Concentration dependence of IAPP aggregation in the absence and presence of lipid membranes. A-D) Normalized ThT fluorescence for IAPP aggregation in the absence of membranes (A) and in the presence of membranes at a 1:10 (B), 1:50 (C) and 1:100 (D) IAPP:lipid molar ratio. The traces in A-D) represent the average with standard deviation of three technical replicates. E) Double log plot of the half time $\left(t_{1 / 2}\right)$ versus the IAPP monomer concentration at $t=0\left(m_{0}\right)$. Scaling exponents $(\gamma$, slope of the line through the datapoints) were derived by linear regression. $\mathbf{F})$ Values of the scaling exponent shown in E) plotted for the different IAPP:lipid ratios.

The scaling exponent can be used to narrow down the possible aggregation mechanisms, which each have a unique dependence on the monomer concentration ${ }^{17}$. We used the online platform AmyloFit to fit our aggregation data to mathematical models representing these different mechanisms ${ }^{17}$. A scaling exponent of -1 may correspond to a simple mechanism consisting of nucleation and elongation, with a reaction order for nucleation $\left(n_{c}\right)$ of 2 . However, this mechanism does not provide a satisfactory fit to our data (Fig. 2A-C). Another possibility is a mechanism incorporating secondary nucleation with a reaction order $\left(n_{2}\right)$ of 1 , which does represent the data well (Fig. 2D-F). The rate constants extracted from these fits reveal that secondary nucleation dominates over primary nucleation by many orders of magnitude (Table S1). Given the relatively minor contribution of primary nucleation, it is difficult to establish the reaction order of this process, with both values of 1 and 2 yielding similarly good fits to the datasets here presented (compare Fig. 2A-C with Fig S2A-C).

Mechanisms with a different scaling exponent, such as secondary nucleation with a reaction order of 2 (theoretically $\gamma=-1.5$ ) or fragmentation $(\gamma=-0.5)^{17}$, do not fit our data as expected (Fig. S2D-I). More complex mechanisms like multi-step secondary nucleation or a combination of secondary nucleation and fragmentation do lead to reasonable fits (Fig. S2J-O). However, these mechanisms should display a curvature in the scaling exponent ${ }^{17}$, which we do not observe within the concentration range used in our experiments (Fig. 1E). 
In sum, the fits suggest that IAPP aggregation on lipid membranes is dominated by secondary nucleation with a reaction order of 1 , corresponding to a critical nucleus size of a single monomer. The addition of fibril seeds strongly reduces the lag phase of IAPP aggregation in the presence of LUVs, providing further support for a secondary nucleation-dominated mechanism ${ }^{17,20,21}$ (Fig. S3).

$1: 10$

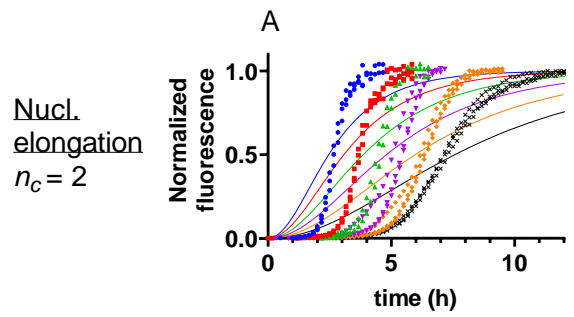

$2^{\circ}$ nucl.

$n_{c}=1$

$n_{2}=1$

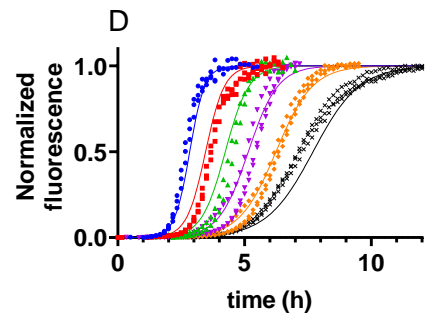

$1: 50$
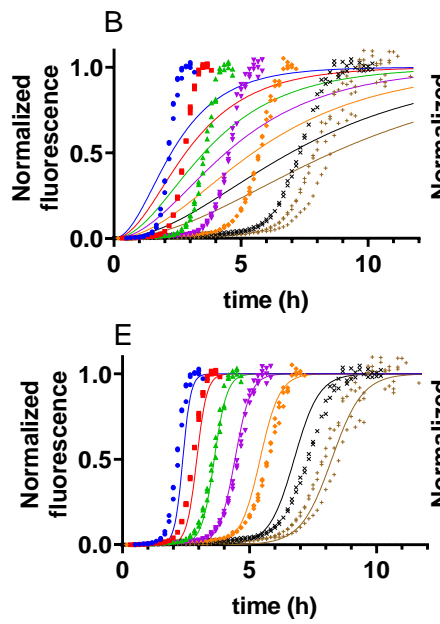

$1: 100$
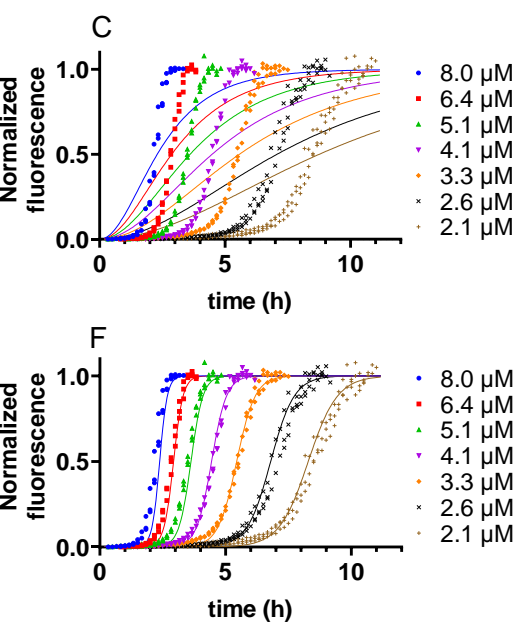

Figure 2) Global fitting of IAPP aggregation kinetics in the presence of LUVs. A, B, C) Fits of ThT aggregation data for a range of IAPP monomer concentrations to a nucleation elongation model with a reaction order for nucleation $\left(n_{c}\right)$ of 2 . D, E, F) Fits of the same data to a model in which secondary nucleation dominates and both reaction orders for primary $\left(n_{c}\right)$ and secondary nucleation $\left(n_{2}\right)$ are equal to 1. Aggregation assays were performed in the presence of 7:3 DOPC:DOPS vesicles at a total IAPP:lipid molar ratio of 1:10 (A, D), 1:50 (B, E) and 1:100 (C, F).

The same aggregation mechanism applies to a range of IAPP:lipid ratios (Fig. 2D-F), in line with the fact that the scaling exponents are very similar (Fig. 1E, Fig. S4A, B). Furthermore, the rate constants extracted from the fits to the 1:50 and 1:100 ratios are in close agreement (Fig. S4C, Table S1). At ratios of 1:10 or lower, we suspect that some IAPP that is not bound to the membrane aggregates in solution, and the scaling exponents and rate constants are essentially a combination of both processes (Fig. S4).

These results are consistent with a critical nucleus size of 1 monomer, in which case the local density of IAPP on the membrane is irrelevant to the nucleation process. To confirm this notion, we performed an additional experiment using a constant concentration of lipids, leading to increased crowding of IAPP on the vesicles with increasing IAPP concentration (Fig. 3A). The scaling exponent and global fitting indeed are consistent with the same mechanism dominated by secondary nucleation with a reaction order of 1 (Fig. 3B-D, Fig. S5). Under these experimental conditions, it becomes evident that a reaction order of 1 is also the preferred solution for primary nucleation (compare Fig. $\mathbf{3 C}$ and Fig. 3D). Altogether, our data indicate that a single IAPP monomer is able to undergo nucleation on the membrane. 

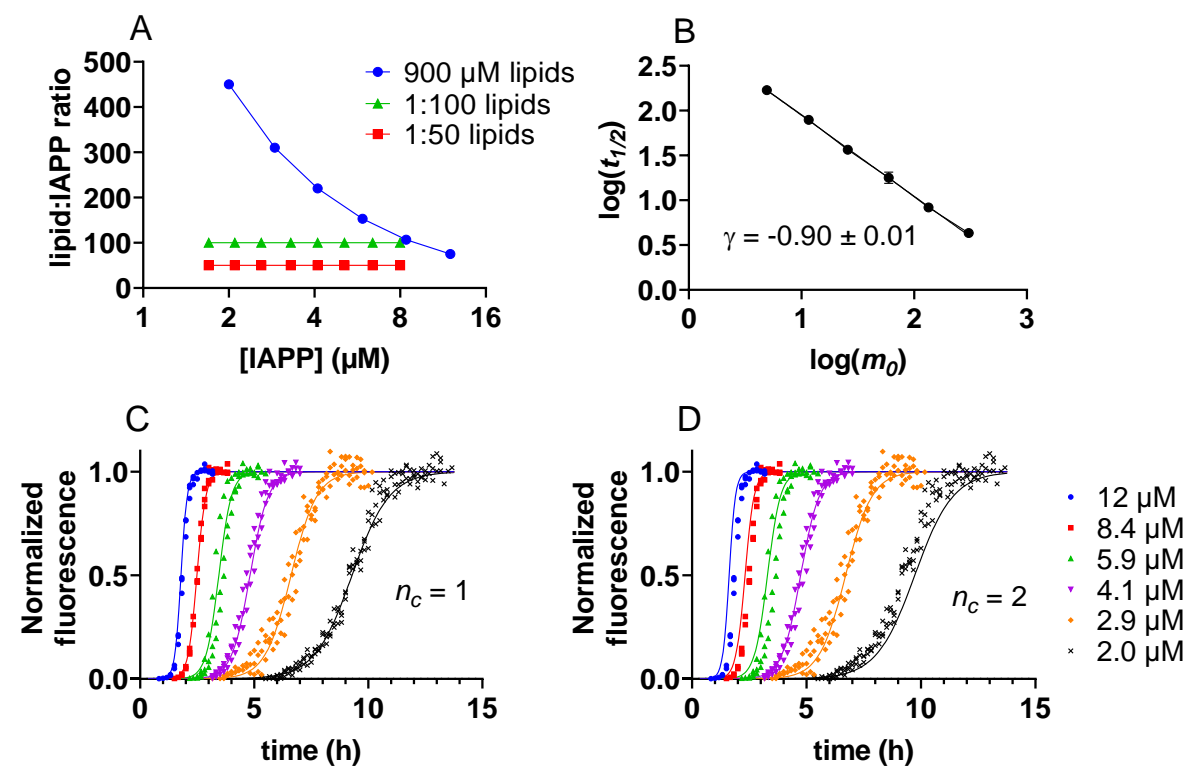

Figure 3) IAPP aggregation at a constant lipid concentration. A) Graphical depiction of the number of lipids for each IAPP molecule at three different experimental conditions: constant lipid concentration (blue) and constant ratios of 1:100 (green) and 1:50 (red). B) Scaling exponent derived from an IAPP aggregation assay performed at a constant lipid concentration of $900 \mu \mathrm{M}$ for all IAPP concentrations. C) ThT fluorescence for IAPP aggregation at a constant lipid concentration of $900 \mu \mathrm{M}$, fitted to a secondary nucleation dominated mechanism. Reaction orders for primary $\left(n_{c}\right)$ and secondary nucleation $\left(n_{2}\right)$ were both set to 1 . D) The same dataset as in C) fitted with $n_{c}=2$ and $n_{2}=1$.

It is well known that anionic lipids speed up the aggregation of $\operatorname{IAPP}^{7,8,11}$. We set out to determine if we could specify which microscopic processes are catalyzed by DOPS. IAPP aggregation assays were performed in the presence of DOPC/DOPS vesicles containing increasing DOPS fractions of $30 \%, 50 \%$ and $70 \%$ (Fig. 4A-C). Increasing the DOPS content dramatically increases the speed of aggregation (note the different $x$-axes in Fig. 4A-C), whereas the scaling exponent remains largely unaffected (Fig. 4D). Thus, the relative abundance of DOPS within the vesicles, but not its total amount increases the catalysis of IAPP aggregation (compare Fig. 4A-C with Fig. 1B-D).

Global fitting of these data (Fig. 4A-C) shows that both the combined rate constants for primary and secondary processes, $k_{+} k_{n}$ and $k_{+} k_{2}$ ( $k_{+}$rate constant for elongation, $k_{n}$ for primary nucleation and $k_{2}$ for secondary nucleation), scale with increasing DOPS fraction (Fig. 4E, Table S1). The fits to unseeded data do not allow the values for $k_{+} k_{n}$ and $k_{+} k_{2}$ to be decomposed into the individual rate constants. To determine whether DOPS promotes nucleation or elongation, we performed strongly seeded experiments in which the initial gradient scales with the elongation rate $k_{+}$(Fig. $\mathbf{4 F}$, see Materials and Methods in Supporting Information). These experiments reveal that elongation is not affected by the DOPS content of the vesicles, and hence the difference in the aggregation kinetics is caused by an increase in the nucleation rates $k_{n}$ and $k_{2}$, with increasing DOPS. With a $\sim 17$-fold increase in $k_{n}$ and $\sim 27$ fold in $k_{2}$ when comparing $30 \%$ with $70 \%$ DOPS, the effect scales more than linearly (Fig. 4E). This finding suggests that the nucleation of one IAPP monomer is stimulated by multiple PS head groups. 
A
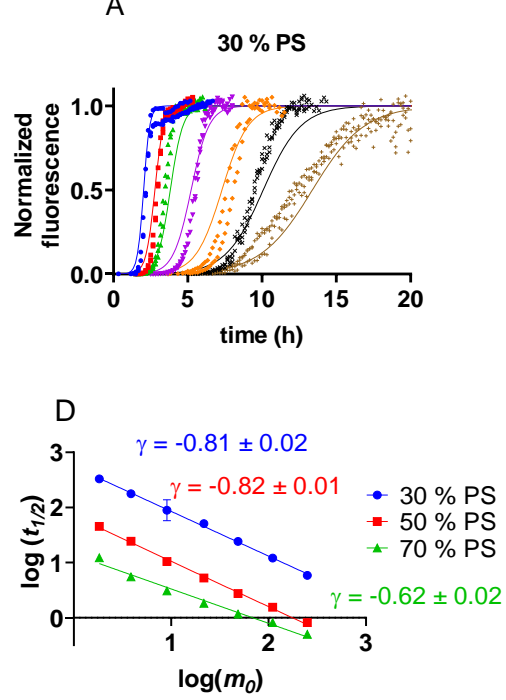

B
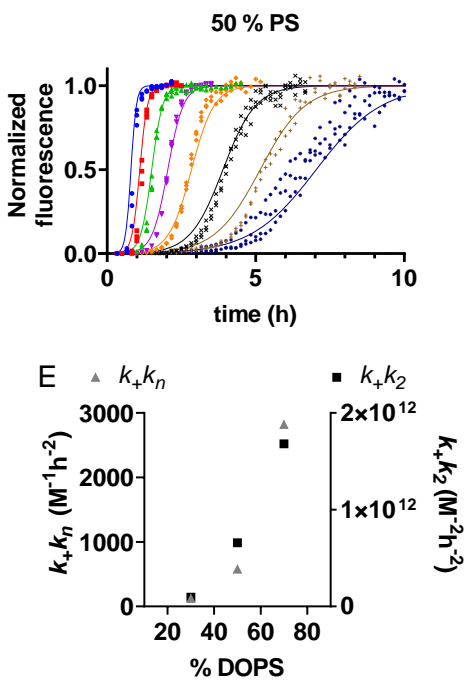

C

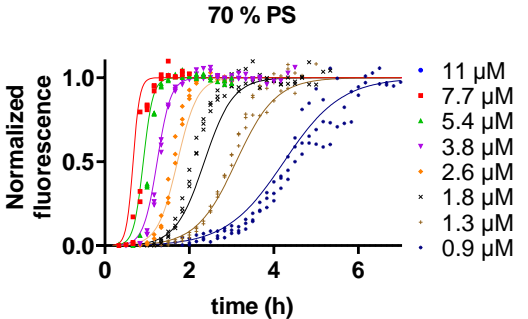

$\mathrm{F}$

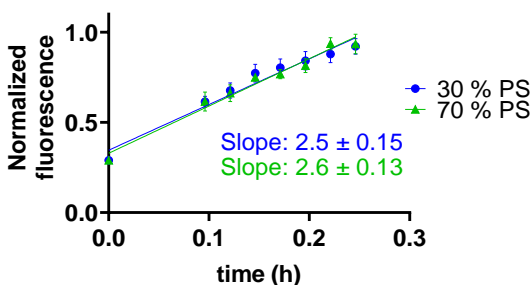

Figure 4) Effect of DOPS on the aggregation kinetics of IAPP. A-C) Normalized ThT fluorescence data of IAPP aggregation in the presence of DOPC/DOPS vesicles with $30 \%$ (A), $50 \%$ (B) and $70 \%$ (C) DOPS content. Data were fitted to a secondary nucleation dominated model with $n_{c}$ and $n_{2}$ of 1 . A total IAPP:lipid ratio of 1:50 was used. D) Scaling exponents of IAPP aggregation in the presence of DOPC/DOPS LUVs with increasing DOPS content. E) Values of the combined rate constants for primary and secondary processes, $k_{+} k_{n}$ and $k_{+} k_{2}$, as a function of DOPS content. F) The initial gradient of a $29 \%$ seeded reaction is similar for both DOPS fractions.

Despite the fact that secondary nucleation has also been reported to dominate IAPP aggregation in solution ${ }^{12-14}$, the aggregation mechanism on lipid membranes is fundamentally different given that it is restricted to a two-dimensional surface. In solution, reaction orders of 2 have been found for the nucleation of IAPP as well as the somewhat similar amyloid- $\beta$ peptide ${ }^{14,16}$. This comparison suggests that the monomeric IAPP nucleus we observe here may be the result of a specific conformation coordinated by DOPS molecules (Fig. 5). The fact that the nucleation rate constants scale more than linearly with the fraction of DOPS suggests that multiple lipid head groups cooperate to induce IAPP nucleation. Furthermore, given that DOPS promotes secondary nucleation, fibril growth must at least partially occur on the membrane surface, in agreement with previous findings for IAPP aggregation ${ }^{22}$ (Fig. 5). By contrast, for amyloid- $\beta$ and $\alpha$-synuclein the addition of lipid vesicles has been reported to promote primary nucleation only ${ }^{23,24}$. Altogether, our results reveal a previously undescribed molecular mechanism of amyloid formation catalyzed by lipid membranes. 


\section{A Primary nucleation}
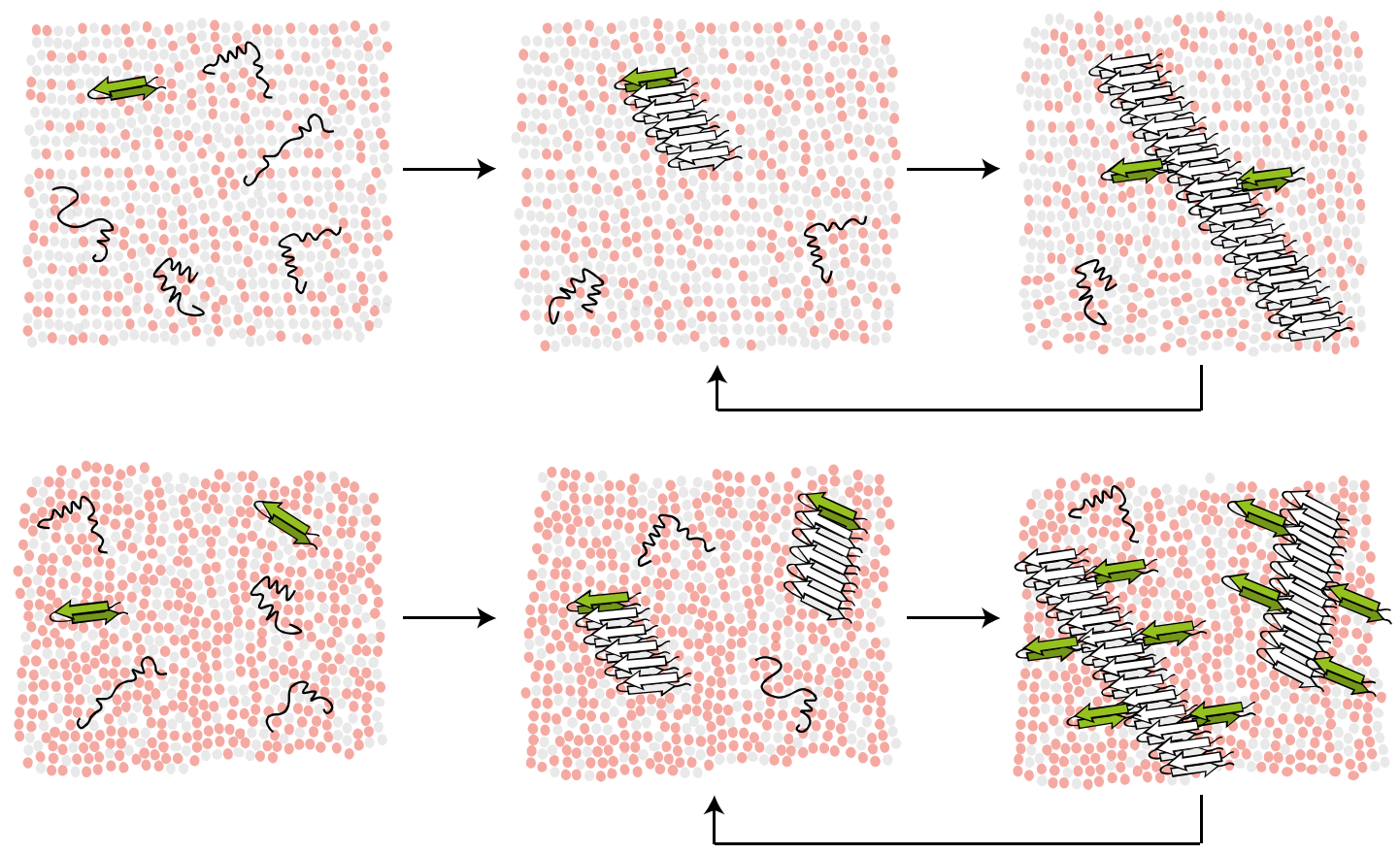

Figure 5) Model for IAPP aggregation catalyzed by membranes containing anionic lipids. A) IAPP can be bound to the membrane in different conformations, and multiple DOPS molecules (red) are involved in the formation of a nucleus (green hairpin). B) Fibril growth occurs at least to some extent on the membrane surface. C) Secondary nucleation occurs at the interface between existing fibrils and DOPS molecules. With increasing DOPS (bottom row), both primary and secondary nucleation are more strongly catalyzed.

\section{Supporting Information}

Materials and Methods, Figures S1-S5 containing supplementary ThT data and additional fits, and Table S1 containing the scaling exponents and rate constants extracted from global fitting.

\section{References}

(1) Bradshaw Kaiser, A.; Zhang, N.; van der Pluijn, W. Global Prevalence of Type 2 Diabetes over the Next Ten Years (2018-2028). Diabetes 2018, 67 (Supplement_1), 202-LB.

(2) Khan, M. A. B.; Hashim, M. J.; King, J. K.; Govender, R. D.; Mustafa, H.; Al Kaabi, J. Epidemiology of Type 2 Diabetes - Global Burden of Disease and Forecasted Trends. J. Epidemiol. Glob. Health 2020, 10 (1), 107-111.

(3) Butler, A. E.; Janson, J.; Bonner-Weir, S.; Ritzel, R.; Rizza, R. A.; Butler, P. C. $\beta$-Cell Deficit and Increased $\beta$-Cell Apoptosis in Humans with Type 2 Diabetes. Diabetes 2003, 52 (1), 102-110.

(4) Yoon, K. H.; Ko, S. H.; Cho, J. H.; Lee, J. M.; Ahn, Y. B.; Song, K. H.; Yoo, S. J.; Kang, M. Il; Cha, B. Y.; Lee, K. W.; et al. Selective $\beta$-Cell Loss and $\alpha$-Cell Expansion in Patients with Type 2 Diabetes Mellitus in Korea. J. Clin. Endocrinol. Metab. 2003, 88 (5), 2300-2308. 
(5) Westermark, P.; Andersson, A.; Westermark, G. T. Islet Amyloid Polypeptide, Islet Amyloid and Diabetes Mellitus. Physiol. Rev. 2011, 91 (3), 795-826.

(6) Milardi, D.; Gazit, E.; Radford, S. E.; Xu, Y.; Gallardo, R. U.; Caflisch, A.; Westermark, G. T.; Westermark, P.; Rosa, C. La; Ramamoorthy, A. Proteostasis of Islet Amyloid Polypeptide: A Molecular Perspective of Risk Factors and Protective Strategies for Type II Diabetes. Chem. Rev. 2021, 121 (3), 1845-1893.

(7) Knight, J. D.; Miranker, A. D. Phospholipid Catalysis of Diabetic Amyloid Assembly. J. Mol. Biol. 2004, 341 (5), 1175-1187.

(8) Jayasinghe, S. A.; Langen, R. Lipid Membranes Modulate the Structure of Islet Amyloid Polypeptide. Biochemistry 2005, 44 (36), 12113-12119.

(9) Engel, M. F. M.; Yigittop, H. A.; Elgersma, R. C.; Rijkers, D. T. S.; Liskamp, R. M. J.; De Kruijff, B.; Höppener, J. W. M.; Antoinette Killian, J. Islet Amyloid Polypeptide Inserts into Phospholipid Monolayers as Monomer. J. Mol. Biol. 2006, 356 (3), 783-789.

(10) Caillon, L.; Lequin, O.; Khemtémourian, L. Evaluation of Membrane Models and Their Composition for Islet Amyloid Polypeptide-Membrane Aggregation. Biochim. Biophys. Acta Biomembr. 2013, 1828 (9), 2091-2098.

(11) Zhang, X.; St Clair, J. R.; London, E.; Raleigh, D. P. Islet Amyloid Polypeptide Membrane Interactions: Effects of Membrane Composition. Biochemistry 2017, 56 (2), 376-390.

(12) Padrick, S. B.; Miranker, A. D. Islet Amyloid: Phase Partitioning and Secondary Nucleation Are Central to the Mechanism of Fibrillogenesis. Biochemistry 2002, 41 (14), 4694-4703.

(13) Patil, S. M.; Mehta, A.; Jha, S.; Alexandrescu, A. T. Heterogeneous Amylin Fibril Growth Mechanisms Imaged by Total Internal Reflection Fluorescence Microscopy. Biochemistry 2011, 50 (14), 2808-2819.

(14) Rodriguez Camargo, D. C.; Chia, S.; Menzies, J.; Mannini, B.; Meisl, G.; Lundqvist, M.; Pohl, C.; Bernfur, K.; Lattanzi, V.; Habchi, J.; et al. Surface-Catalyzed Secondary Nucleation Dominates the Generation of Toxic IAPP Aggregates. Front. Mol. Biosci. 2021, 8, 757425.

(15) Knowles, T. P. J.; Waudby, C. A.; Devlin, G. L.; Cohen, S. I. A.; Aguzzi, A.; Vendruscolo, M.; Terentjev, E. M.; Welland, M. E.; Dobson, C. M. An Analytical Solution to the Kinetics of Breakable Filament Assembly. Science 2009, 326 (5959), 1533-1537.

(16) Cohen, S. I. A.; Linse, S.; Luheshi, L. M.; Hellstrand, E.; White, D. A.; Rajah, L.; Otzen, D. E.; Vendruscolo, M.; Dobson, C. M.; Knowles, T. P. J. Proliferation of Amyloid-B42 Aggregates Occurs through a Secondary Nucleation Mechanism. Proc. Natl. Acad. Sci. U. S. A. 2013, 110 (24), 9758-9763.

(17) Meisl, G.; Kirkegaard, J. B.; Arosio, P.; Michaels, T. C. T. T.; Vendruscolo, M.; Dobson, C. M.; Linse, S.; Knowles, T. P. J. J. Molecular Mechanisms of Protein Aggregation from Global Fitting of Kinetic Models. Nat. Protoc. 2016, 11 (2), 252-272.

(18) Brender, J. R.; Krishnamoorthy, J.; Sciacca, M. F. M.; Vivekanandan, S.; D'Urso, L.; Chen, J.; La Rosa, C.; Ramamoorthy, A. Probing the Sources of the Apparent Irreproducibility of Amyloid Formation: Drastic Changes in Kinetics and a Switch in Mechanism Due to Micellelike Oligomer Formation at Critical Concentrations of IAPP. J. Phys. Chem. B 2015, 119 (7), 28862896.

(19) Dada, S. T.; Hardenberg, M. C.; Mrugalla, L. K.; Mckeon, M. O. Spontaneous Nucleation and Fast Aggregate-Dependent Proliferation of $\alpha$-Synuclein Aggregates within Liquid Condensates 
at Physiological PH. BioRxiv 2021.

(20) Cohen, S. I. A.; Vendruscolo, M.; Dobson, C. M.; Knowles, T. P. J. From Macroscopic Measurements to Microscopic Mechanisms of Protein Aggregation. J. Mol. Biol. 2012, 421 (23), 160-171.

(21) Arosio, P.; Cukalevski, R.; Frohm, B.; Knowles, T. P. J.; Linse, S. Quantification of the Concentration of $A \beta 42$ Propagons during the Lag Phase by an Amyloid Chain Reaction Assay. J. Am. Chem. Soc. 2014, 136 (1), 219-225.

(22) Engel, M. F. M.; Khemtémourian, L.; Kleijer, C. C.; Meeldijk, H. J. D.; Jacobs, J.; Verkleij, A. J.; De Kruijff, B.; Killian, J. A.; Höppener, J. W. M. Membrane Damage by Human Islet Amyloid Polypeptide through Fibril Growth at the Membrane. Proc. Natl. Acad. Sci. U. S. A. 2008, 105 (16), 6033-6038.

(23) Habchi, J.; Chia, S.; Galvagnion, C.; Michaels, T. C. T.; Bellaiche, M. M. J.; Ruggeri, F. S.; Sanguanini, M.; Idini, I.; Kumita, J. R.; Sparr, E.; et al. Cholesterol Catalyses A 42 Aggregation through a Heterogeneous Nucleation Pathway in the Presence of Lipid Membranes. Nat. Chem. 2018, 10 (6), 673-683.

(24) Galvagnion, C.; Buell, A. K.; Meisl, G.; Michaels, T. C. T.; Vendruscolo, M.; Knowles, T. P. J.; Dobson, C. M. Lipid Vesicles Trigger $\alpha$-Synuclein Aggregation by Stimulating Primary Nucleation. Nat. Chem. Biol. 2015, 11, 229-234. 